\title{
Etude des fluctuations d'intensité issue d'une fibre optique multimodale à gradient d'indice couplée partiellement avec une surface détectrice circulaire
}

\author{
I. Verrier et J. P. Goure \\ Laboratoire Traitement du Signal et Instrumentation, UA-CNRS 842, Université de St-Etienne, Faculté des \\ Sciences et Techniques, 23, rue du Docteur-Paul-Michelon, 42023 St-Etienne Cedex 2, France
}

(Reçu le 25 juillet 1986, accepté le 31 octobre 1986)

\begin{abstract}
Résumé. - L'intensité issue de la section d'une fibre optique à gradient d'indice de courte longueur convoluée par une surface de même dimension est évaluée ainsi que le rapport signal à bruit. Les résultats théoriques et expérimentaux concordent pour une fibre en mouvements aléatoires et éclairée en lumière incohérente. La fibre étant au repos, on observe une discontinuité du rapport signal à bruit en lumière cohérente comme en lumière incohérente.
\end{abstract}

\begin{abstract}
The intensity at the end face of a short graded-index optical fiber is evaluated in a convolution by a same size area in the same way as the signal to noise ratio. The theoretical and experimental results with an incoherent light are in good agreement when the fibre randomly vibrates. When the fibre is motionless, the signal to noise ratio shows a rough variation for a coherent light as well as for an incoherent one.
\end{abstract}

\section{Introduction.}

Le flux issu de l'extrémité d'une fibre optique multimodale éclairée par une source lumineuse présente des fluctuations liées aux différents modes de propagation de la fibre et à la source. La parfaite connaissance de ces phénomènes est importante dans le cas de mesure de puissance lumineuse, ces fluctuations du système donnant une imprécision sur le résultat. L'étude statistique de ce phénomène a été effectuée par plusieurs auteurs en lumière cohérente et en particulier pour des fibres à saut d'indice [1-7]. Ils ont évalué le rapport signal à bruit et la fonction de densité de probabilité de puissance. L'analyse de la granularité a aussi été effectuée à l'aide d'une barrette de photodiodes [1]. Le cas du couplage d'une fibre avec un détecteur de même axe et de diamètre variable a été envisagé $[2-4,7]$. Dans le cas d'une fibre à gradient d'indice, les variations de la figure de speckle en fonction du mésalignement du faisceau incident ont par ailleurs été étudiées expérimentalement [8] ainsi que celles dues à un mésalignement entre deux fibres [9]. Dans ce dernier cas, le contraste est mesuré en fonction de la distance $r$ au centre sur l'embout de sortie de la fibre à l'aide d'une méthode de champ proche.

Par opposition aux précédents travaux, nous nous sommes intéressés non pas à l'énergie et au contraste mesurés à partir du centre de la fibre de sortie, mais à l'énergie contenue dans l'intersection de deux surfaces identiques dont les axes sont décalés progressivement et au rapport signal à bruit correspondant. Ces deux surfaces représentent soit les cœurs d'une fibre à gradient parabolique et d'une fibre à saut d'indice, soit le cœur d'une fibre et d'un détecteur.

Dans une première partie, nous calculons l'énergie transmise en lumière incohérente dans le couplage d'une fibre à gradient d'indice avec une surface décalée et de même dimension. Nous décrivons ensuite la méthode expérimentale utilisée. Nous comparerons enfin les résultats théoriques et expérimentaux.

\section{Calcul de la convolution.}

On cherche à calculer l'énergie transmise dans l'intersection des deux surfaces représentant par exemple l'embout d'une fibre à gradient d'indice et la surface d'un détecteur (Fig. 1). On suppose que le profil d'indice de la fibre est parabolique. La relation de Gloge et Marcatili [10] permet alors d'évaluer l'intensité moyenne dans le temps $\langle I(r, t)\rangle$ en fonction de la distance $r$ au centre de la fibre en champ proche :

$$
\langle I(r, t)\rangle=\frac{\left\langle P_{0}\right\rangle}{2 \pi} \cdot\left(\frac{V}{a}\right)^{2}\left[1-g\left(\frac{r}{a}\right)\right]
$$


avec

$$
g\left(\frac{r}{a}\right)=\left(\frac{r}{a}\right)^{2}
$$

où $a$ est le rayon du cœur de la fibre, $\left\langle P_{0}\right\rangle$ dépend de la source, $V$ est la fréquence normalisée qui s'exprime en fonction du vecteur d'onde $k_{0}$ et des indices au centre du cœur $n_{1}$ et de gaine $n_{2}$

$$
V=a \cdot k_{0} \cdot n_{1} \sqrt{2 \Delta} ; \quad \Delta=\frac{n_{1}^{2}-n_{2}^{2}}{2 \cdot n_{1}^{2}}
$$

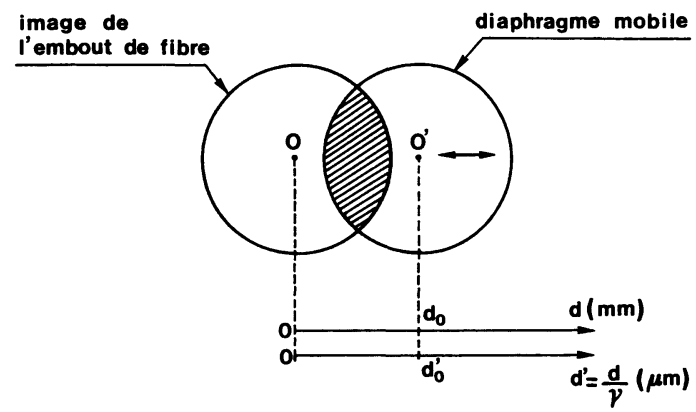

Fig. 1. - Représentation dans le plan image D de l'intersection de la tache et du diaphragme. La partie hachurée contient l'énergie couplée, $d$ est la position du diaphragme, $d^{\prime}$ le défaut de couplage, $\gamma$ le grandissement.

[Representation in the plane D of image and diaphragm intersection. The hatching part contains the coupled energy, $d$ is the diaphragm location, $d^{\prime}$ is the coupling fault, $\gamma$ is the magnitude.]

On peut alors calculer le produit de convolution $\left\langle X\left(d^{\prime}, t\right)\right\rangle$ en fonction de la distance $d^{\prime}$ entre les deux centres $\mathrm{O}$ et $\mathrm{O}^{\prime}$ :

$$
\begin{aligned}
\left\langle X\left(d^{\prime}, t\right)\right\rangle=2 \int_{d^{\prime}-a}^{a} \operatorname{Arccos} & \left(\frac{r^{2}+d^{\prime 2}-a^{2}}{2 r \cdot d^{\prime}}\right) \times \\
& \times\langle I(r, t)\rangle \cdot r \cdot \mathrm{d} r .
\end{aligned}
$$

$\left\langle X\left(d^{\prime}, t\right)\right\rangle$ représente l'énergie transmise dans la région hachurée sur la figure 1 . Ce résultat n'est valable que si la source est incohérente car on raisonne sur les intensités. Le calcul de cette intégrale est donné en annexe. On trouve en posant :

$$
\begin{array}{r}
u=d^{\prime} / 2 a \quad \text { et } \quad B=\frac{\left\langle P_{0}\right\rangle}{\pi}\left(\frac{V}{a}\right)^{2} \\
\frac{\langle X(u, t)\rangle}{B a^{2}}=\frac{1}{4} \operatorname{Arccos} u+u\left(u^{2}+\frac{1}{2}\right) \sqrt{1-u^{2}}+ \\
+2\left(\frac{1}{8}-u^{2}\right)\left[\operatorname{Arcsin}(-u)+\frac{\pi}{2}\right] .
\end{array}
$$

On peut évaluer le rapport signal à bruit en supposant que les fluctuations ne portent que sur $P_{0}$. Dans ces conditions, la variance $\sigma^{2}$ s'écrit :

$$
\begin{aligned}
& \sigma^{2}=\left\langle X\left(d^{\prime}, t\right)^{2}\right\rangle-\left\langle X\left(d^{\prime}, t\right)\right\rangle^{2} \\
& \sigma^{2}=\frac{V^{4}}{\pi^{2} a^{4}} Y^{2}\left[\left\langle P_{0}^{2}\right\rangle-\left\langle P_{0}\right\rangle^{2}\right]
\end{aligned}
$$

avec

$$
\begin{aligned}
Y=\int_{d^{\prime}-a}^{a} \operatorname{Arccos}\left(\frac{r^{2}+d^{\prime 2}-\alpha^{2}}{2 r \cdot d^{\prime}}\right) \times \\
\times\left[1-\left(\frac{r}{a}\right)^{2}\right] \cdot r \cdot \mathrm{d} r .
\end{aligned}
$$

Le rapport signal à bruit est alors donné par :

$$
\frac{\mathrm{S}}{\mathrm{B}}=\frac{\left\langle P_{0}\right\rangle}{\left\{\left\langle P_{0}^{2}\right\rangle-\left\langle P_{0}\right\rangle^{2}\right\}^{1 / 2}} .
$$

Ce rapport est donc indépendant de $d^{\prime}$. Ceci est vérifié s'il y a équilibre des modes.

\section{Dispositif expérimental.}

L'appareillage utilisé est décrit figure 2. L'embout d'une fibre optique est éclairé par une source lumineuse, soit directement, soit à travers un objectif de microscope $(20 \mathrm{X}$, ouverture numérique 0,4$)$. Celle-ci est un laser $\mathrm{He}-\mathrm{Ne}$ polarisé avec ou sans polariseur ou une source de lumière blanche. On a utilisé un mètre

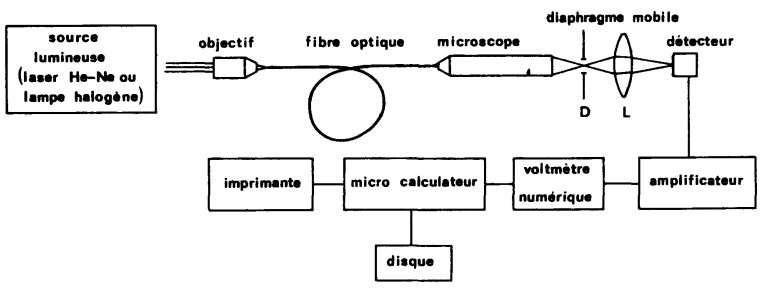

Fig. 2. - Montage expérimental.

[Experimental set-up.]

d'une fibre multimodale C.L.T.O. G3B 77 A/6 de $50 \mu$ de cœur et d'ouverture numérique 0,224 . On forme l'image de la face de sortie à l'aide d'un microscope de grandissement $\gamma$ (objectif 63X, oculaire 10X) dans un plan $\mathrm{D}$. Un diaphragme circulaire de même diamètre $(16,5 \mathrm{~mm})$ que la tache image est translaté d'une distance $d$ dans $\mathrm{D}$ perpendiculairement au plan de la figure 2. La tache image correspond uniquement au cœur de la fibre. Contrairement à certaines fibres, la lumière de gaine a complètement disparu au bout d'une longueur de un mètre de ce type de fibre. Ceci a été contrôlé à l'aide de photographie des embouts de fibre sur le même montage. L'énergie issue de l'intersection de l'image et du diaphragme est recueillie sur une lentille de grand diamètre et focalisée sur un détecteur large $\left(5,1 \mathrm{~mm}^{2}\right.$ EG\&G HUV $\left.1000 \mathrm{~B}\right)$. Le signal envoyé par celui-ci est transmis à un voltmètre numérique puis à un micro-calculateur. Les résultats sont traités et stockés sur disquette. La température est maintenue constante à l'intérieur du laboratoire.

Pour chaque position $d$ du diaphragme, on enregistre un grand nombre de points de mesure (minimum 750) toutes les $90 \mathrm{~ms}$. Le voltmètre intègre, sur $6,6 \mathrm{~ms}$, neuf 
échantillons qui correspondent à un seul point de mesure. On calcule ensuite la valeur moyenne dans le temps de la tension de sortie $\langle X(d, t)\rangle$ du détecteur et son écart-type $\sigma$. Le rapport signal à bruit pour la tension mesurée est calculé à l'aide de la relation :

$$
\frac{\mathrm{S}}{\mathrm{B}}=\frac{\langle X(d, t)\rangle}{\sigma}=\frac{\langle X(d, t)\rangle}{\left[\left\langle X(d, t)^{2}\right\rangle-\langle X(d, t)\rangle^{2}\right]^{1 / 2}}
$$

La tension délivrée par le système de détection est proportionnelle au flux lumineux. Par suite, le rapport signal à bruit pour l'énergie suit la même loi.

La procédure utilisée permet ainsi de simuler expérimentalement le défaut de couplage latéral $d^{\prime}$ d'une
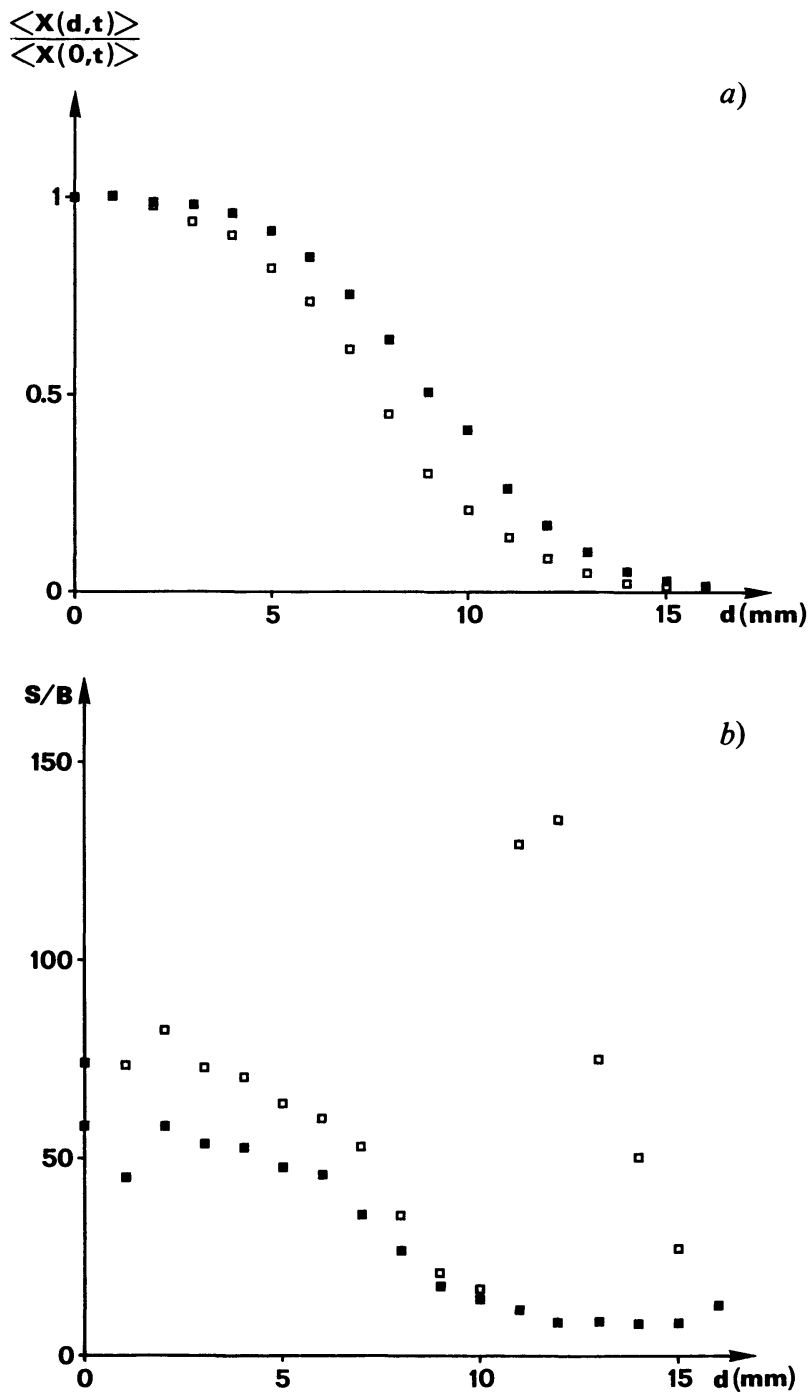

Fig. 3. - Source He-Ne et polariseur : a) Variation de la valeur moyenne normée $\langle X(d, t)\rangle /\langle X(0, t)\rangle$ en fonction de $d$. b) Variation du rapport signal à bruit en fonction de $d$. $\square$ sans mouvements de la fibre ; $\square$ avec mouvements de la fibre.

[He-Ne laser and polarizer: a) Normalized mean value $\langle X(d, t)\rangle /\langle X(0, t)\rangle$ versus $d$. b) Signal to noise ratio versus $d . \square$ in the case of motionless; $\square$ with fibre vibrations.] fibre soit avec une fibre à saut d'indice de même ouverture numérique, soit avec un détecteur (Fig. 1). Une expérience de couplage direct fibre-fibre nécessiterait une précision mécanique bien supérieure à celle requise dans ce montage.

\section{Résultats.}

4.1 LUMIÈRE COHÉRENTE. - La première source utilisée est constituée du laser polarisé He-Ne suivi d'un polariseur permettant d'atténuer le faisceau. Celui-ci est focalisé sur la face d'entrée de la fibre. Les mesures effectuées ont montré que le système étant immobile, la valeur moyenne $\langle X(d, t)\rangle$ décroît régulièrement lorsque $d$ augmente (Fig. 3a) et par contre le rapport signal à bruit subit d'abord une décroissance, puis une brusque et importante remontée (Fig. 3b).

Lorsqu'on soumet la fibre à des mouvements aléatoires, pour les différentes positions $d$ du diaphragme, on observe une valeur moyenne $\langle X(d, t)\rangle$ plus faible que dans le cas précédent et décroissant plus lentement en fonction de $d$ (Fig. 3a). Le rapport signal à bruit ne présente plus la discontinuité précédente et diminue constamment avec $d$ (Fig. 3b).

Cette brusque variation du signal à bruit, lorsque la fibre est au repos, est indépendante des conditions d'injection. L'utilisation du laser focalisé en avant de la fibre (Fig. 4a) ou du laser sans objectif conduit au même résultat (Fig. 4b). Ceci permet de penser que les modes à fuite n'interviennent pas et que tous les modes guidés sont excités.

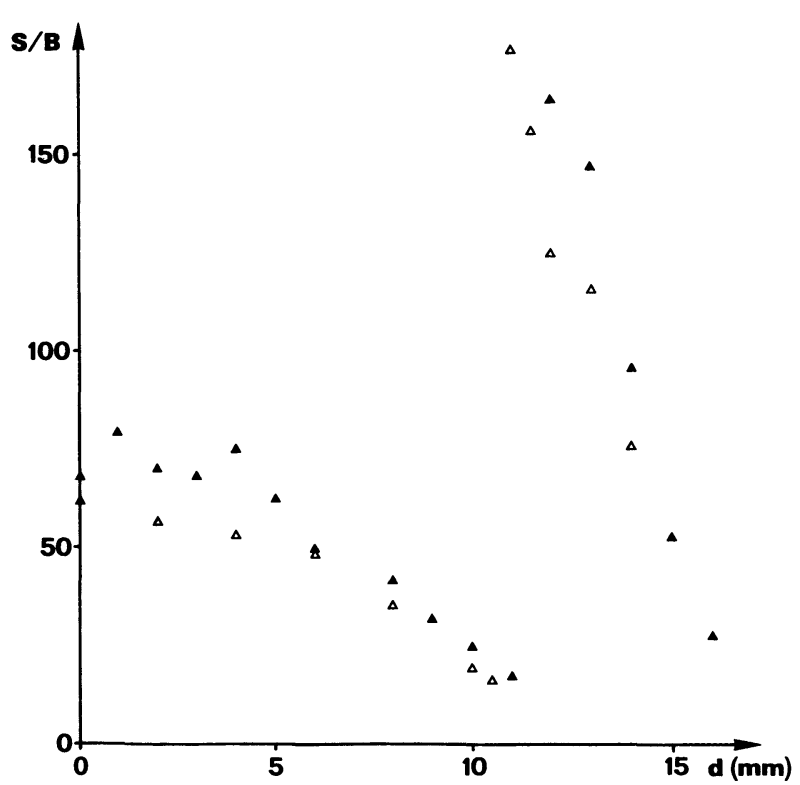

Fig. 4. - Variation du rapport signal à bruit en fonction de $d$ lorsque la fibre est au repos : a) $\Delta$ laser focalisé en avant de la fibre ; b) $\Delta$ laser sans objectif.

[Plot of signal to noise ratio against $d$ when the fibre is motionless : a) $\Delta$ laser focused forward the fibre end face ; b) $\Delta$ laser without objective.] 
4.2 LUMIÈRE INCOHÉRENTE. - Une source incohérente focalisée sur la fibre est utilisée ensuite comme émetteur. Si la fibre subit des mouvements, la courbe $\langle X(d, t)\rangle$ expérimentale (Fig. 5) coïncide avec la courbe théorique donnée par la relation (4) et le rapport signal à bruit mesuré varie de manière aléatoire autour d'une valeur moyenne (Fig. 6) ; ceci confirme donc que celui-ci est indépendant de $d$ comme nous l'avons déjà signalé.

Lorsque la fibre est au repos, la valẹur moyenne du signal $\langle X(d, t)\rangle$ conserve les mêmes valeurs, cependant la courbe du rapport signal à bruit, en fonction de $d$, présente une remontée importante pour une distance $d_{0}$ comme pour la source cohérente (Fig. 6).

4.3 Discussion. - L'analyse de l'ensemble des résultats obtenus avec le laser montre que la rupture observée sur les courbes signal à bruit pour une fibre au repos est toujours située à la distance $d_{0}$ qui correspond à la chute de l'écart-type $\sigma$ sur la mesure $X(d, t)$ (Fig. 7).

Si $a$ est le rayon de cœur de la fibre, cette distance $d_{0}$ est reliée à un rayon $r_{0}$ de la fibre par :

$$
r_{0}=\frac{d_{0}}{\gamma}-a=d_{0}^{\prime}-a
$$

$\left(d_{0}^{\prime}:\right.$ distance ramenée à la grandeur du cœur de la fibre).

Le phénomène observé montre une fluctuation plus

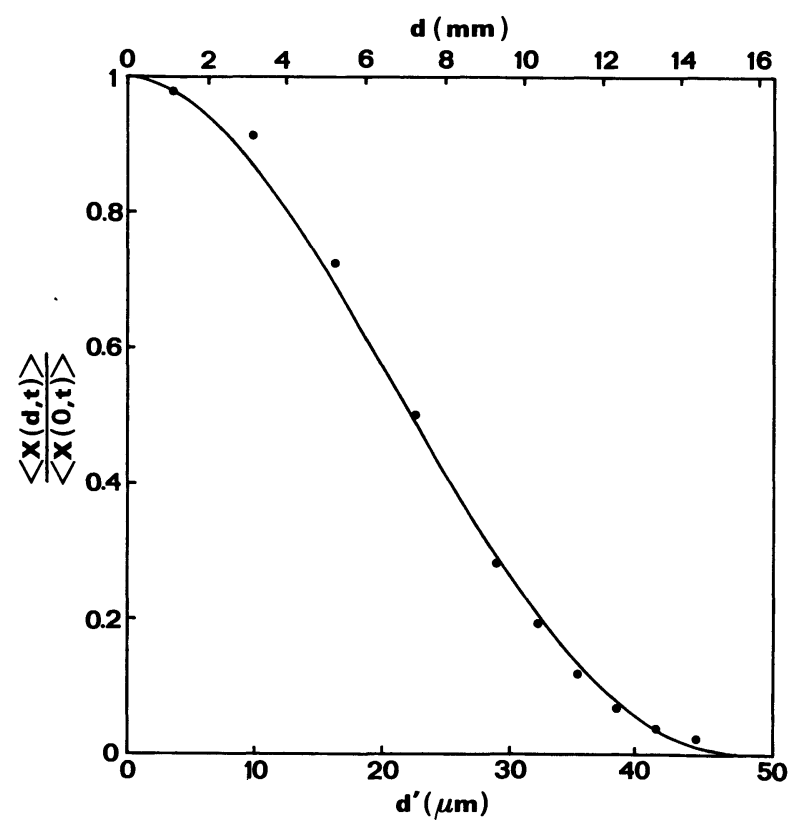

Fig. 5. - Source incohérente: Variation de la valeur moyenne normée $\langle X(d, t)\rangle /\langle X(0, t)\rangle$ : • en fonction de $d$ pour les valeurs expérimentales; - en fonction de $d^{\prime}$ pour les valeurs théoriques.

[Incoherent light. Plot of the normalized mean value $\langle X(d, t)\rangle /\langle X(0, t)\rangle$ : • against $d$ for experimental data ; — against $d^{\prime}$ for theoretical values.]

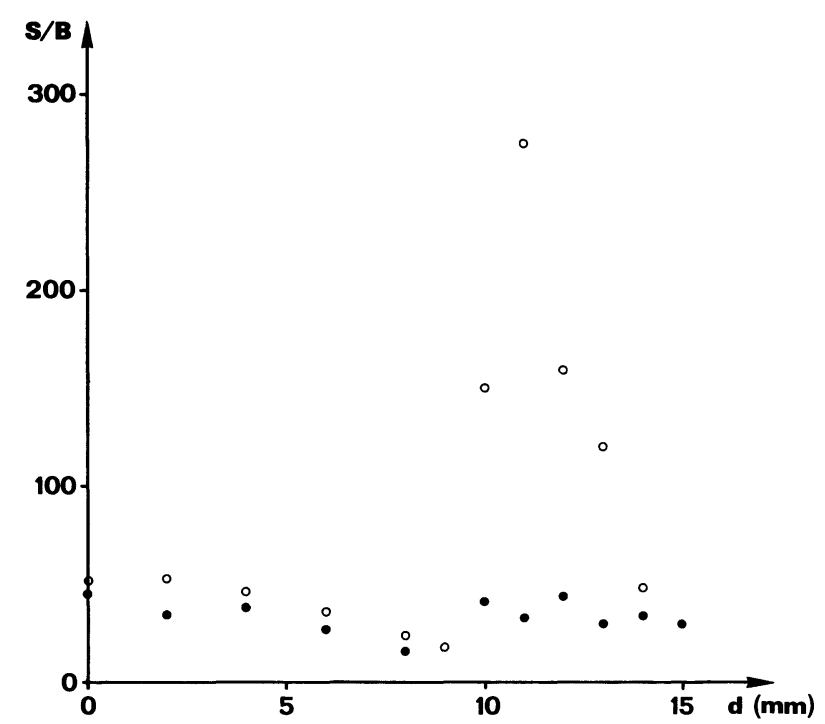

Fig. 6. - Variation du rapport signal à bruit en lumière incohérente : $O$ sans mouvements de la fibre ; • avec mouvements de la fibre.

[Plot of signal to noise ratio in incoherent light. $O$ in the case of motionless fibre ; $\bullet$ with fibre vibrations.]

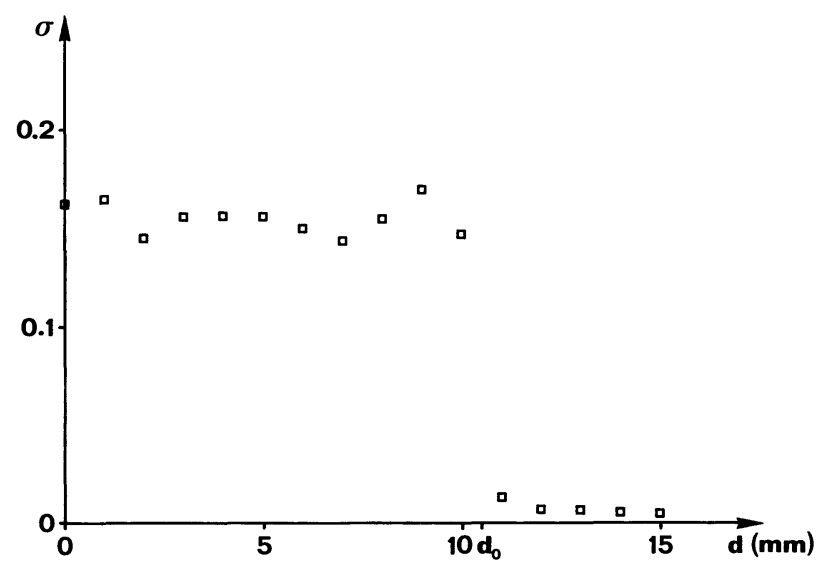

Fig. 7. - Variation de l'écart-type $\sigma$ sur $X(d, t)$ en fonction de $d$ en lumière cohérente.

[Plot of the standard deviation $\sigma$ on $X(d, t)$ against $d$ in coherent light.]

importante de l'énergie pour $r<r_{0}<a$. Par contre, pour $r>r_{0}$, l'énergie est plus faible, mais fluctue beaucoup moins (Fig. 7 et Fig. 3a). Le rapport signal à bruit redécroît uniquement parce que la valeur moyenne $\langle X(d, t)\rangle$ diminue avec $r, \sigma$ restant fixe. Une des causes de ce phénomène est une fluctuation plus importante dans l'énergie des modes d'ordre les plus bas qui transportent la part la plus grande de l'énergie. On retrouve un résultat bien connu : l'équilibre en énergie n'est pas réalisé pour ceux-ci sur une si courte longueur de fibre. Seuls les modes les plus hauts semblent stabilisés, les modes près de l'axe fluctuant beaucoup. 
La mise en mouvement de la fibre en lumière cohérente conduit à des valeurs du rapport signal à bruit et de la valeur moyenne différentes des résultats précédents car le mélange de modes est meilleur. Cependant, nous n'arrivons pas à obtenir un rapport signal à bruit constant car subsistent toujours des interférences entre modes (Fig. 3b).

En lumière incohérente, si on soumet la fibre à des mouvements aléatoires, nous arrivons à un équilibre et nos calculs de convolution s'ajustent alors très bien à nos valeurs expérimentales (Fig. 5). En laissant la fibre au repos, nous ne pouvons pourtant pas atteindre une stabilisation des modes (Fig. 6).

Puisque la discontinuité du rapport signal à bruit est observée en lumière cohérente et en lumière incohérente, les fluctuations ne sont pas seulement dues aux oscillations du laser HeNe. D'autre part, elles ne sont pas induites par une influence de la température maintenue stable. Toutefois pour un centrage parfait $(d=0)$, les fluctuations de la puissance totale du laser seul (sans fibre) restent importantes. Elles se superposent à celles apportées par les mouvements propres de la fibre pour induire du bruit modal. La mise en mouvement de la fibre conduit à un lissage de la courbe du rapport signal à bruit plus ou moins important suivant la nature de la source.

\section{Conclusion.}

Dans cet article, nous avons étudié théoriquement et expérimentalement, l'intensité issue de la section d'une fibre optique à gradient d'indice de courte longueur, couplée à une surface désalignée de même dimension.

La méthode utilisée permet de simuler expérimentalement un défaut de couplage avec un détecteur ou une fibre à saut d'indice.

Si nous soumettons la fibre à des mouvements aléatoires en lumière incohérente, le rapport signal à bruit devient proche de la valeur théorique que nous attendions et la courbe $\langle X(d, t)\rangle$ correspond au produit de convolution calculé.

L'étude a également montré que le rapport signal à bruit du flux mesuré sur le cœur de la fibre de courte longueur, sans mouvements, présente une forte discontinuité pour un défaut de couplage déterminé quelle que soit la nature de la source.

Tous ces résultats confirment que les fluctuations de puissance optique en transmission dépendent de la fibre et du couplage.

Dans le cas d'un émetteur avec fibre amorce multi-• mode, en l'absence de mélangeur de modes (ou mouvements de la fibre), la présence de celle-ci engendre des fluctuations même si la source est stabilisée et même si on réalise un bon couplage.

\section{Annexe}

Calcul de $\left\langle X\left(d^{\prime}, t\right)\right\rangle$

$$
\begin{gathered}
\left\langle X\left(d^{\prime}, t\right)\right\rangle=2 \times \int_{d^{\prime}-a}^{a}\left[\operatorname{Arccos}\left(\frac{r^{2}+d^{\prime 2}-a^{2}}{2 r \cdot d^{\prime}}\right)\right] I(r) \cdot r \cdot \mathrm{d} r \\
I(r)=\frac{B}{2}\left(1-\frac{r^{2}}{a^{2}}\right) ; B=\frac{P_{0}}{\pi}\left(\frac{V}{a}\right)^{2} \\
\left\langle X\left(d^{\prime}, t\right)\right\rangle=B \times\{\underbrace{\left.\int_{d^{\prime}-a}^{a}\left[\operatorname{Arccos}\left(\frac{r^{2}+d^{\prime 2}-a^{2}}{2 r \cdot d^{\prime}}\right)\right] r \cdot \mathrm{d} r-\int_{d^{\prime}-a}^{a}\left[\operatorname{Arccos}\left(\frac{r^{2}+d^{\prime 2}-a^{2}}{2 r \cdot d^{\prime}}\right)\right] \frac{r^{3}}{a^{2}} \mathrm{~d} r\right\} .}_{J_{1}} \underbrace{}_{J_{2}}
\end{gathered}
$$

Ces deux intégrales s'intègrent par parties en posant : $v=\operatorname{Arccos}\left(\frac{r^{2}+d^{\prime 2}-a^{2}}{2 r d^{\prime}}\right) \quad$ et $\quad w=\left\{\begin{array}{lll}r & \text { pour } & J_{1} \\ r^{3} & \text { pour } & J_{2}\end{array}\right.$

$J_{1}$ et $J_{2}$ se décomposent alors à l'aide d'autres intégrales de la forme :

$I_{m}=\int_{d^{\prime}-a}^{a} \frac{r^{m-1}}{m} \times \frac{\mathrm{d} r}{\sqrt{\frac{4 r^{2} a^{2}}{\left(r^{2}-d^{\prime 2}+a^{2}\right)^{2}}-1}} \quad m=2,4$

On effectue alors le changement de variable :

$$
r^{2}=u^{\prime}+d^{\prime 2}-a^{2} \text { et } v^{\prime}=\frac{u^{\prime}-2 a^{2}}{2 a d^{\prime}}
$$

$J_{1}$ et $J_{2}$ sont alors des combinaisons linéaires d'intégrales $K_{n}$ de la forme :

$$
K_{n}=\int_{-1}^{-d^{\prime} / 2 a} \frac{v^{\prime n} \mathrm{~d} v^{\prime}}{\sqrt{1-v^{\prime 2}}} \quad n=0,1,2
$$

qui s'intègrent immédiatement :

$$
\begin{aligned}
& K_{0}=\left[\operatorname{Arcsin} v^{\prime}\right]_{-1}^{d^{\prime} / 2 a} \\
& K_{1}=\left[-\sqrt{1-v^{\prime 2}}\right]_{-1}^{-d^{\prime} / 2 a} \\
& K_{2}=\left[-\frac{v^{\prime}}{2} \sqrt{1-v^{\prime 2}}+\frac{1}{2} \operatorname{Arcsin} v^{\prime}\right]_{-1}^{-d^{\prime} / 2 a} .
\end{aligned}
$$


On obtient alors :

$$
\frac{\left\langle X\left(d^{\prime}, t\right)\right\rangle}{B}=\left[\frac{r^{2}}{2}\left(1-\frac{r^{2}}{2 a^{2}}\right) \operatorname{Arccos}\left(\frac{r^{2}+d^{\prime 2}-a^{2}}{2 r \cdot d^{\prime}}\right)\right]_{d^{\prime}-a}^{a}-\frac{d^{\prime}}{4 a}\left(d^{\prime 2}+a^{2}\right) K_{1}+\left(\frac{a^{2}-d^{\prime 2}}{4}\right) K_{0}-\frac{d^{\prime 2}}{2} \cdot K_{2}
$$

et :

$$
\frac{\left\langle X\left(d^{\prime}, t\right)\right\rangle}{B \cdot a^{2}}=\frac{1}{4} \operatorname{Arccos}\left(\frac{d^{\prime}}{2 a}\right)+\frac{d^{\prime}}{2 a}\left(\frac{d^{\prime 2}}{4 a^{2}}+\frac{1}{2}\right) \sqrt{1-\frac{d^{\prime 2}}{4 a^{2}}}+2\left(\frac{1}{8}-\frac{d^{\prime 2}}{4 a^{2}}\right) \cdot\left[\operatorname{Arcsin}\left(-\frac{d^{\prime}}{2 a}\right)+\frac{\pi}{2}\right] .
$$

\section{References}

[1] Rawson, E. G. and Goodman, J. W., S.P.I.E., Applications of speckle phenomena, 1980, vol. $243,28$.

[2] Daino, B., de Marchis, G., Piazzola, S., Electron. Lett. 15 (1979) 755.

[3] Rawson, E. G., Goodman, J. W., Norton, R. E., Opt. Lett. 5 (1980) 357.

[4] Goodman, J. W., Rawson, E. G., Opt. Lett. 6 (1981) 324.

[5] Couch, P. R., Epworth, R. E., J. Lightwave Technol. LT 1 (1983) 591.
[6] Kanada, T., J. Lightwave Technol. LT 2 (1984) 11. [7] Daino, B., De Marchis, G., Piazzola, S., Opt. Acta 27 (1980) 1151.

[8] Tsuji, T., Asakura, T., FujI, H., Opt. Quantum Electron. 16 (1984) 9.

[9] Tsuji, T., Asakura, T., FujII, H., Opt. Quantum Electron. 16 (1984) 197.

[10] Gloge, D., Marcatili, E., Bell Syst. Tech. J. 52 (1973) 1563. 